\title{
DAM and interoperable DRM: Maintaining agility in the world of evolving content ecosystems
}

\section{Gord Larose}

has been a DRM technology expert from long before DRM was a viable business. A professional engineer and CISSP (Certified Information Systems Security Professional), Gord maintains the online "DRM Dictionary" and is the author of numerous publications and patents in the field.

Keywords: digital rights management, digital asset management, interoperability, content ecosystem, metadata, rights expression language

\begin{abstract}
The current situation in digital rights management (DRM), particularly DRM interoperability, is described as it relates to the field of digital asset management (DAM). In particular the emergence of a few large "content ecosystems" which have their own specific attributes in DRM and other areas such as business rules is described. While full interoperability of these ecosystems at the consumer level may not happen for many years, interoperability of key back-end infrastructure such as DAM systems is possible and desirable now. A proposal is put forward on how DAM systems should evolve architecturally in such a way as to maximize both their scope of application and their level of content security - and thus their value. It is argued that such evolution is valuable from a product agility perspective even for DAM systems which do not interact with the world of consumer media.
\end{abstract}

\section{EXECUTIVE SUMMARY}

Over the last decade or so the technologies of digital asset management (DAM) and digital rights management (DRM) have both evolved in response to the challenges of managing and monetizing large amounts of online digital content. It is obviously appropriate in some situations for DAM and DRM systems to work hand-in hand, or even be integrated. Integrating DAM with DRM is complicated by the existence of several highly successful but incompatible "content ecosystems" such as the Windows Media Player ecosystem - which have incompatible DRM technologies.

Work is ongoing within the DRM community on interoperability efforts which have the potential to bridge these DRM systems. These efforts may take years to bear fruit, and in any event, are aimed at interoperability primarily from the consumer viewpoint, so they are unlikely to help the integration of DAM and DRM. Because DAM systems interact with the back-ends of these ecosystems, and because the ecosystems are already partially metadata-driven, however, DAM interoperability with multiple ecosystems is now achievable.

Providing such interoperability - and the architectural flexibility required to deliver it - has numerous benefits. The DAM systems which are most agile, and 
which demonstrably address content owners' security concerns, will participate most in the huge and expanding markets of online consumer content. Even for DAM vendors not contemplating the consumer mass market, the same architectural flexibility would be of benefit in adapting to their own evolving (eg enterprise) markets.

\section{THE INTEROPERABILITY CHALLENGE}

Recent literature has described how DAM systems help with the business of content management in general, ${ }^{1}$ and how DAM technology can be integrated with DRM technology. ${ }^{2}$

Unfortunately, there is not, practically speaking, one kind of DRM technology for DAM (or any other related technology) to integrate with. This is clearly apparent in the consumer arena through the battle between Apple's iTunes, Microsoft's Windows Media Player, and the Open Mobile Alliance's OMA-DRM. Each of these co-existing "content ecosystems" has its own DRM technology, user base and business rules.

This paper focuses primarily on the scenario of a DAM technology provider that wishes to participate in these ecosystems. Clearly, not all DAM providers may wish to do so; some are well-positioned within vertical enterprise markets based on technologies such as Windows Rights Management System (RMS). It is argued, however, that these ecosystems provide a vast market opportunity, and further, that almost any DAM system could benefit from the same architectural evolution as is presented for this scenario.

Today's content ecosystems interoperate only in very limited ways, and there is increasing pressure for interoperability. Initiatives such as the Coral Consortium ${ }^{3}$ are addressing the issue of end-to-end interoperability, notably including interoperability at the level of individual consumer devices. For many reasons - commercial, legal and technical - providing interoperable DRM down to the consumer level is extremely challenging. Some observers feel it may take many years. It is tempting to sit on the sidelines and watch this play out before making any decisions.

From a DAM perspective, however, sitting on the sidelines is a bad idea. It is not necessary to wait and see whether consumer-level interoperability comes to pass, because DAM systems do not operate at the consumer level. Most DAM systems live implicitly at the periphery of one DRM ecosystem and/ or one content provider, with little agility to link new content providers to new ecosystems or vice versa. The argument for DAM interoperability is therefore a simple one of market reach and cost effectiveness, which is not dependent on consumer-level interoperability.

The challenge, then, is to make DAM technology agile enough to interact seamlessly with multiple content sources and multiple evolving content ecosystems, which differ in DRM and other areas. The general nature of the solution is clear - to make increasing use of metadata and context-driven functions, as opposed to expensive custom coding. Today's DRM ecosystems do not have enough in common to support a "big bang" improvement where one DAM system could interwork with all of them. DAM system designers and users therefore face a phased evolution - one which 
requires crucial architectural decisions regarding which points of flexibility they incorporate first.

Further, if the DAM community wishes to become entrenched in these profitable ecosystems, it may be time for them to adopt some of the ecosystems' security concepts. This includes in particular the concept of "robustness" that applications not only enforce appropriate security, even for insiders, but also resist skilled attempts to modify them to break the rules. Given the sensitive position of some DAM systems within the content chain, it is in the interests of content managers to make sure that, for example, a single disgruntled employee cannot - even if he is a skilled hacker - suddenly cause the music of his favorite band to be distributed as "public domain."

\section{BACKGROUND}

Until recent years, DRM was a struggling field providing a solution to problems most people didn't know they had. In the 1990s, many small companies offering DRM technology solutions came and went. Many ran out of capital before they could gain traction in a market that was reasonably content with existing, physical methods of content distribution. Indeed, as anyone who has read The Innovator's Dilemma ${ }^{4}$ will appreciate, it was precisely the large, profitable, incumbent content owners who had no interest in new distribution channels or the DRM that such channels required. The "two guys in a garage" shops with no money were more interested, but were hardly the ideal customers for a start-up company looking for quick revenue growth.

The reluctance of established content providers to get on the DRM bandwagon was, in hindsight, quite understandable. At the time, DRM solutions were mostly proprietary, point technologies from small start-up companies. They may have solved the content protection problem quite well, but content providers were understandably reluctant to commit their content to such systems. For one thing, content providers did not feel capable of accurately evaluating the security of the various solutions pitched to them. For another, they were reluctant to deal with small start-ups which had a significant risk of failing and leaving them with "orphan" content and unsupportable technology. Finally, content providers at the time faced many other pieces of the puzzle, which DRM technology providers did not appreciate, or address. Notable among these were the questions of content licensing, business models, online distribution and content management.

DRM was first seen in the 1990s, although the term DRM was not usually applied at the time:

- copy protection in VCRs proved a commercial boon to Macrovision through the royalty stream generated by mandated inclusion of the technology in millions of VCRs;

- the PC and video console game markets generated a wide variety of anti-copy schemes in an attempt to preserve revenue by reducing piracy, notably in expensive "first-person shooter" games;

- the Content Scrambling System (CSS) which (however poorly) protected the MPEG-2 video content of DVD movie disks.

In hindsight, this era provided the 
following lessons to the content and DRM communities:

- it is difficult just to manage and distribute vast amounts of content, even without trying to protect it - this provided impetus to the DAM market;

- any content protection scheme can probably be cracked - although this in itself does not make content protection a bad idea;

- economic success for a DRM provider has at least as much to with do business and political savvy and corporate credibility, as it does with the quality of the technology;

- online content providers hate having to deal with many unrelated solutions which only address part of the problem, and/or start-up companies which may not be around for the long term.

\section{THE DOMINANCE OF CONTENT AND DRM ECOSYSTEMS}

Major players in the DRM landscape today, such as Apple and Microsoft, were happy to watch on the sidelines as DRM start-ups came and went before the turn of the millennium. In the new millennium, they realized that the time was right and created major DRMprotected content ecosystems of their own design. Of course, Apple and Microsoft didn't think of themselves as creating ecosystems, but the metaphor is apt. iTunes and Windows Media are huge distributed systems involving millions of items of content, tens of millions of endusers, dozens of software applications and thousands of network nodes. The vision is the same for the Open Mobile Alliance's OMA-DRM technology, although consumers may be less familiar with it.

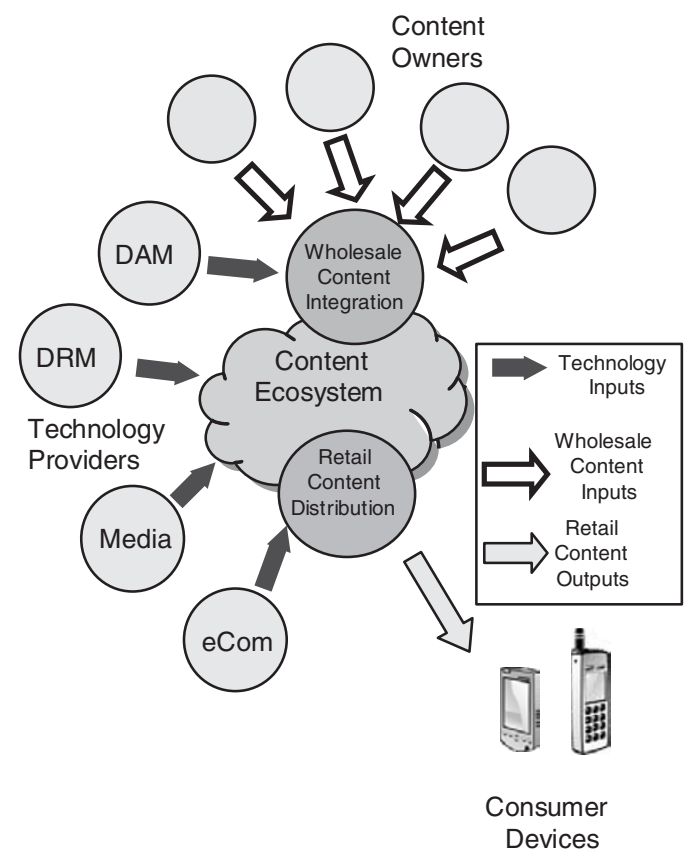

Figure 1: A content ecosystem in context

Figure 1 illustrates a typical content ecosystem in the context of its major inputs and outputs. On the left are the technology suppliers who provide the technology infrastructure components - from codecs to DRM to DAM which are required to keep the ecosystem running. On the top are the various content owners - such as music studios - who, it is hoped, will contribute their content to this ecosystem.

One of the ways to induce content owners to do so is by ensuring that they do not need to worry about technical details in general and content security (ie DRM) in particular. The ecosystem architecture should do this. In the case illustrated, the "wholesale content integration" component - which is to a large extent a DAM system — performs this hiding function.

Content security is the primary raison d'etre of DRM. DRM ecosystems have rules about the ecosystem's behavior to 
which all participants in the ecosystem must adhere. These rules are enforced through licensing agreements and other means including, in the worst case, revocation (disabling) of noncompliant components such as consumer players. As a result, content providers can be quite confident that, while breaches may occur, the overall ecosystem will protect their content in the long term.

Such rules come in two main forms: compliance rules and robustness rules. Compliance rules ensure that components of the ecosystem behave in accordance with the applicable licensing terms for content in the ecosystem; for example, that a free sample song to be played only for one day expires the next day. Robustness rules ensure that components of the ecosystem resist attempts by hackers to bend or break the foregoing adherence to compliance rules; for the foregoing example, to ensure that the sample song cannot be "cracked" for unlimited free play. Robustness rules cannot literally prevent a device from being hacked, but they do make it more difficult, and by conforming to such rules, a manufacturer demonstrates due diligence which is typically a licensing requirement for becoming part of the ecosystem.

Currently, these rules are primarily directed at end-user devices (whether software or hardware based) such as portable media players. This is the best place to start introducing such rules, because they are readily accessible and exist in great numbers in public hands, thereby presenting the largest security risk. Ecosystem owners have expressed the intention to keep "raising the bar," not just in increasing security expectations for access devices, but also in the scope of the ecosystem components to which the rules apply. For example, there are also compliance rules and robustness rules which apply to the production of content on back-end systems which are different from, but similar in spirit to, the rules for its rendering on portable devices. These rules are not written with DAM systems in mind - yet - but those responsible for DAM systems, which are currently viewed as peripheral to these ecosystems, will sooner or later, as they become more closely allied with such systems, find themselves accountable for the compliance and robustness of their DAM applications.

There are differences between the DRM employed by different ecosystems in general, and these rules in particular, but these are not a significant differentiator between ecosystems for most industry players. A content provider or device manufacturer is more likely to choose an ecosystem to participate in based on business factors. First among these, at least for manufacturers, is whether the ecosystem accepts outside members. The dominant iTunes ecosystem is largely closed to anyone outside Apple and is considered to be an ecosystem only because it is so large. To pursue the biological metaphor a step further, it is more of a monoculture. (Indeed, Apple does not externally publish robustness rules, because they develop all the code in question themselves.) Windows Media Player and OMA are the most significant other ecosystems, and they actively court third-party participation in order to help gain critical mass in the marketplace.

Table 1 describes the key characteristics of these three major 
Table 1: Core content ecosystems

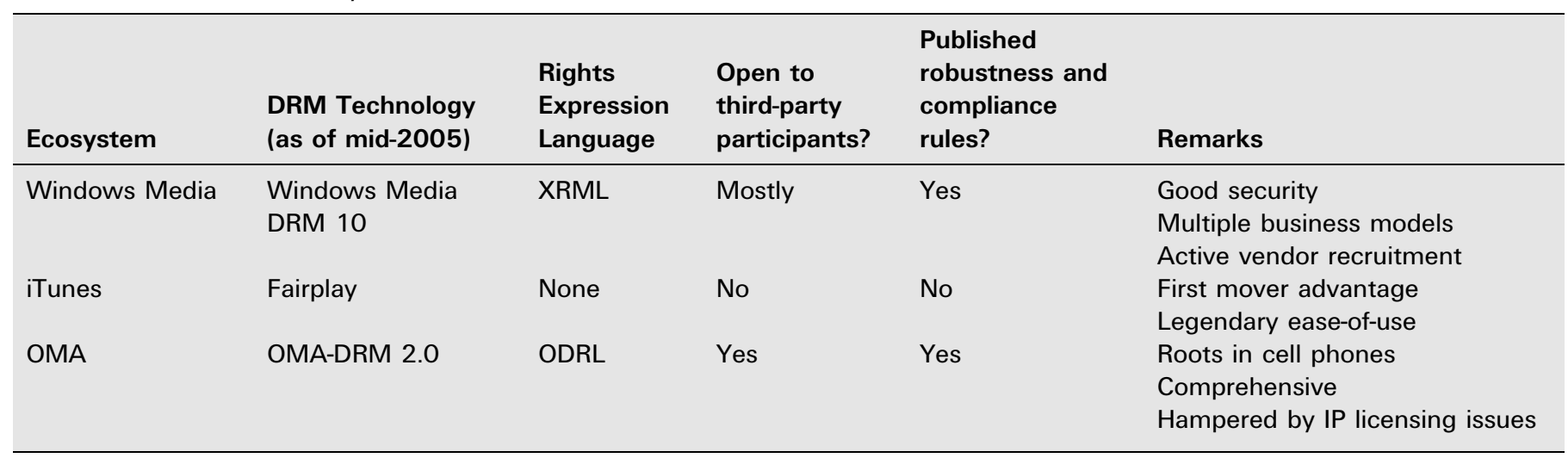

content ecosystems in the market today.

In addition to these big three core ecosystems, there are several smaller standards-driven content handling domains which deserve mention. These do not compete with the core ecosystems, but sometimes interact with them at the edges. They are link protocols which ensure that content which is already licensed and distributed is handled securely between multiple devices at the consumer level, for example, between a PC and a television within one household.

Other than as yet one more possible source of metadata, these link protocols are unlikely to directly affect DAM infrastructure in the near future. It is nonetheless useful for DAM providers to understand them, so as to be able to distinguish such cases from core ecosystems as both continue to evolve in the future.

Typical of these is the Digital Transmission Content Protection over Internet Protocol (DTCP-IP), which is used to protect high-definition television signals streamed over a multivendor inhome network. Others include High bandwidth Digital Content Protection (HDCP) and Windows Media Connect.
The key things to bear in mind about these peripheral domains are:

- Technology providers aren't free to copy content from a core ecosystem to a link protocol "just because they can," any more than they are free to copy content from, say, iTunes to Windows Media. There are negotiated policies covering the interaction with these protocols, which may add per-title metadata which a DAM system must manage and protect. For example, there is a negotiated agreement between Microsoft and the licensing body for DTCP-IP which permits sending certain Windows Media content across DTCP-IP links assuming that DTCP-IP "copy control" bits are set properly.

- Content providers must consider these link protocols from a security point of view and should ensure that they don't provide new material weaknesses in content protection.

\section{FITTING THE ECOSYSTEMS TOGETHER}

To illustrate how DAM and various DRM ecosystems interact, let us take the scenario of a DAM technology provider looking to establish or expand 
its presence in the online consumer entertainment market. The opportunity is illustrated in Figure 2. If a DAM system can enable the flexible "wholesale content integration" function in the middle of this diagram, it can interact with both multiple content providers and multiple content ecosystems. This provides a scalable growth opportunity for the enabling DAM technology, which does not depend on solving the truly difficult problem of interoperating right down to the consumer device level.

What does that technology provider need to do to its technology to fit in?

In a word: abstraction. Hard-coding must be phased out in favor of metadata-driven design. At least two distinct flavors of metadata are required.

There is, of course, the explicit metadata associated with the individual media files being managed, some of it

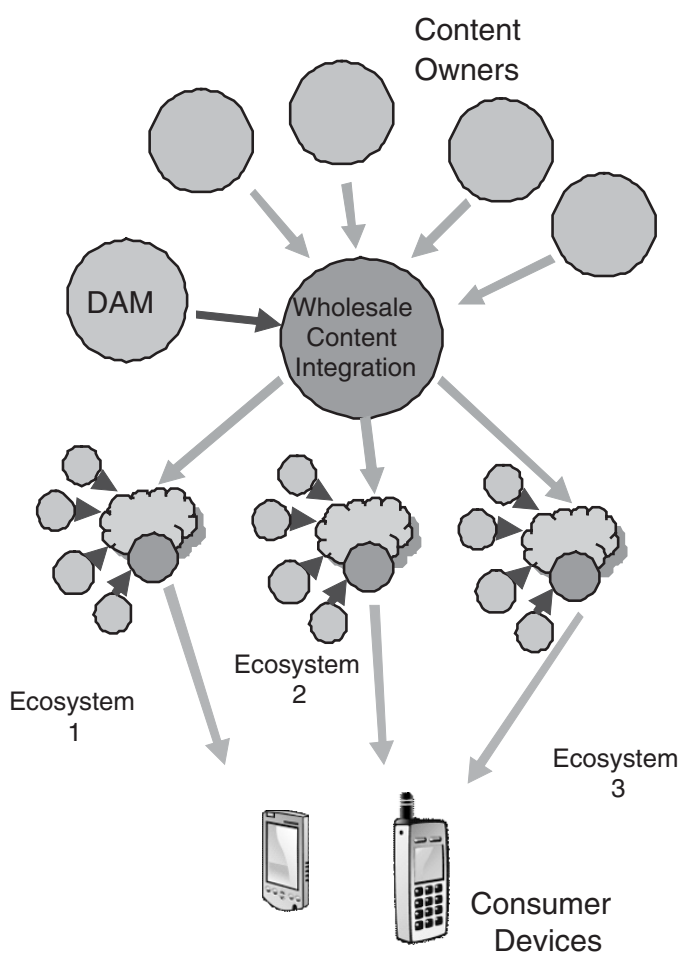

Figure 2: DAM interworking with multiple ecosystems rights metadata and some of it routine information such as title, artist and so forth. Rights metadata are captured in a rights expression language (REL). The leading REL is eXtensible Rights Markup Language (XRML), which Microsoft's ecosystem uses. XRML is also the basis for Motion Picture Expert's Group (MPEG) rights management work, although this may be moot since no major ecosystem is MPEG-based. The second-place REL is Open Digital Rights Language (ODRL), due to its selection as the REL for the OMA's DRM system. Rights metadata are not universal; for example, the leading iTunes ecosystem has no explicit rights metadata.

A good DAM design, therefore, must treat the existence and type of content metadata as a form of "meta-meta" data in itself. The design must also consider to what extent content metadata are simply "passed through" from a content provider vs created or modified by the DAM system. In most cases, content providers will only specify basic business and security parameters for a title. It will be up to other infrastructure components such as DAM systems to use templates to build the required detailed metadata for each applicable ecosystem.

Finally, of course, the DAM system must protect this content metadata, preferably even from inside attacks. Attacks on this metadata could result in large-scale deployment of inadequately protected or unprotected content. A good way to approach this problem is to choose a specific pair of compliance rules and robustness rules from a content ecosystem, and apply them to your design. It doesn't matter too much which specific set you choose, because 
they are all quite similar in spirit. They are not usually very prescriptive, which gives you flexibility in how security is attained. In addition, referring to such a pair of rule sets would be a great way to convince key ecosystem participants such as content providers that you understand content security.

The second kind of metadata is required from an operations point of view, to provide the flexible data flow and data transformations required to manage the implied many-to-many relationships. Where does a particular content provider's content come from? Which ecosystems does each content provider's content feed into? What are the default policies for each such combination? How are per-title exceptions and similar special cases handled? Is the DAM required to transform these policies into usable form such as content licenses or metadata, or are some of those operations performed by other components of each ecosystem?

Note that in this scenario - unlike a vertical business DAM situation - the rights that the organization using the DAM system has to the content may be completely different, requiring yet another set of metadata. Or it may be, as an intermediary, that the organization actually has no direct rights to the content.

\section{CONCLUSIONS}

Several powerful, convergent trends have profoundly changed the nature of the content business over the last several years. Digital media technologies have made it possible for any consumer to get any content, any where, any time.

DRM technologies have emerged to ensure that consumers have, at least most of the time, both the opportunity and the incentive to acquire legitimate rights for the content they use. DAM technologies help content owners and distributors deal with managing large amounts of online content. That content is often restricted to a vertical enterprise, but there is good reason to construct DAM systems to support the wider world of consumer content.

The online content business has, at least in the consumer arena, coalesced into a small number of large "ecosystems," each of which has its own approach to many requirements, from distribution methods to business models to DRM. In practice, therefore, "DRM interoperability" as such, is too narrow a goal; it is the overall ecosystems which must interoperate, in DRM and other aspects. As most of these ecosystems are economically desirable distribution channels, most content providers will choose to work with several of them at once.

In order for DAM systems to be the technology of choice for managing content in this situation, they must be inherently agile. Not only must they understand the DRM requirements of major ecosystems, but also their business rules, distribution methods and back-end processes. Most of these attributes are not currently described in any abstract fashion such as an XML vocabulary; rather, they are typically hard-coded into DAM systems today. Even where such abstract descriptions are supported, such as in the case of rights expression languages, there are at least two different standards, and many domains don't use a standard REL at all.

Cementing the role of DAM systems in this new world requires the following: 
- an understanding of content ecosystems and their key interfaces;

- identifying the specific ecosystems which are significant to you and your clients today, and mapping the common and divergent requirements to your implementation;

- designing DAM applications to provide concrete, designed-in reassurances about compliance and robustness by adopting the concepts (and perhaps the specifics) of compliance rules and robustness rules from the DRM community;

- ensuring the right points of flexibility are integrated into your evolving design so that the next change that comes along can be handled with metadata and plug-in code, and not significant rewrites.

\section{References}

1 Marcus, I. (2005) "The DAM vendor landscape: What the buyer should know" Journal of Digital Asset Management, Vol. 1 No. 1 , pp. 46-58.

2 Rosenblatt, B. and Dykstra, G. (2003) "Integrating content management with digital rights management", May.

3 The Coral Consortium: http:// www.coral-interop.org.

4 Christensen, C. M. (1997) The Innovator's Dilemma. Harvard Business School Press. 